Bundesgesundheitsbl 2015 $\cdot 58: 515-518$

DOI 10.1007/s00103-015-2139-0

Online publiziert: 5. Mai 2015

c) Springer-Verlag Berlin Heidelberg 2015

\author{
Arne Simon ${ }^{1} \cdot$ Hansjürgen Piechota ${ }^{2} \cdot$ Martin Exner $^{3} \cdot J^{\prime}$ oachim Martius ${ }^{4}$ \\ ${ }^{1}$ Klinik für Pädiatrische Onkologie und Hämatologie, Universitätsklinikum \\ des Saarlandes, Homburg/Saar, Deutschland \\ 2 Johannes Wesling Klinikum, Minden, Deutschland \\ ${ }^{3}$ Universitätsklinikum Bonn, Bonn, Deutschland \\ ${ }^{4}$ Gynäkologische Abteilung, Krankenhaus Agatharied, Hausham, Deutschland
}

\title{
Katheterassoziierte Harnwegsinfektionen - neue KRINKO-Empfehlung zur Prävention
}

- durch die Berücksichtigung alternativer Möglichkeiten, wie der intermittierenden Selbst- oder Fremdkatheterisierung [3], sowie

- durch die Begrenzung der Liegedauer von HWK $[4,5]$.

Hierzu gibt es eine Reihe von Studien, in denen ein durch das Pflegepersonal umgesetztes Standardprotokoll zur frühzeitigen Entfernung jedes Blasenkatheters führte, der nicht mehr benötigt wurde [6-10].

Voraussetzung dafür ist eine abteilungsspezifische, dem Behandlungsteam bekannte Liste von medizinischen Indikationen für die Anlage eines Blasenverweilkatheters. Tatsächlich können die Details dieser Indikationen in Abhängigkeit vom jeweiligen Fachgebiet und der individuellen Behandlungssituation der Patienten erheblich variieren. So kann zum Beispiel bei einem Patienten in der Neurochirurgie eine instabile Wirbelkörperfraktur die Indikation für einen Harnwegskatheter darstellen [10]. Bei onkologischen Patienten mit hämorrhagischer Zystitis (nach Ifosfamid-Therapie oder bei einer BK-Virus-Infektion der Harnblase nach Stammzelltransplantation) [11] kann die Anlage eines Spülkatheters indiziert sein.

In einer US-amerikanischen Studie wussten $41 \%$ der zuständigen Ärzte nicht, dass ihr Patient einen Harnwegskatheter hatte und bei $31 \%$ aller HWK lag keine begründete medizinische Indikation vor [12]. In anderen Studien wurden vergleichbar hohe oder noch höhere Raten für die medizinisch nicht indizierte Anwendung von HWK gefunden [13-17].

Nach übereinstimmenden Ergebnissen von zwei systematischen Übersichtsarbeiten zu den verfügbaren Studien [18, 19] führen Erinnerungssysteme zu einer verkürzten Liegedauer von HWK und zu einer reduzierten CA-UTI-Rate. Solche Erinnerungssysteme (reminder) sollen Krankenpflegepersonal und die behandelnden Ärzte zu definierten Zeitpunkten dazu bewegen, die Indikation für den HWK täglich aufs Neue zu überprüfen [5, 20].

Vor allem in der postoperativen Intensivtherapie werden HWK oft zu lange belassen. Meist kann ein HWK bereits nach 48-72 h wieder entfernt werden, wenn keine anderslautende ärztliche Verordnung hierzu vorliegt. Entgegen weit verbreiteter Meinung hatte die Senkung der Anwendungsrate für HWK auf einer neurochirurgischen Intensivstation keinen signifikanten Einfluss auf die Inzidenz von Dekubiti; lediglich Dekubiti im Windelbereich der Schweregrade III und IV wurden in dieser Studie als medizinisches Argument für die Anwendung einer dauerhaften Katheterdrainage der Harnblase zur Förderung der Wundheilung akzeptiert [10]. 


\section{Harnwegsinfektionen leitliniengerecht diagnostizieren und therapieren}

Nosokomiale Harnwegsinfektionen verlaufen in den meisten Fällen nicht lebensbedrohlich. Ihre Behandlung erfordert jedoch - auch wenn sie leitliniengerecht und gezielt erfolgt - den Einsatz von Antibiotika, wodurch der Selektionsdruck im Krankenhaus weiter ansteigt. Heute wird nicht mehr wie noch vor einigen Jahren zur Surveillance bei allen Intensivpatienten mit Harnwegskatheter in regelmäßigen Abständen eine Urinprobe in die Mikrobiologie geschickt [21], die dann je nach Liegedauer des Katheters mehrheitlich eine signifikante Bakteriurie (z. B. mit $>10^{3} \mathrm{KBE} / \mathrm{ml}$ einer einzelnen Spezies) ergibt. Auch eine Pyurie mit positiver Leukozytenesterase im Streifentest kann bei Patienten mit Harnwegskatheter vorkommen, ohne dass eine Harnwegsinfektion vorliegt [22]. Die Unterscheidung der in diesem Kontext nahezu regelhaften asymptomatischen Bakteriurie von einer symptomatischen, behandlungsbedürftigen Harnwegsinfektion bereitet im klinischen Alltag immer noch Schwierigkeiten [23]. Darauf weisen zahlreiche Studien hin, die einen exzessiven Einsatz von Antibiotika „zur Therapie von Harnwegsinfektionen“ darstellen, obwohl in der Mehrzahl der Fälle definitiv keine Harnwegsinfektion vorlag [24-26].

Von allen Patienten mit einer harnwegskatheterassoziierten Bakteriurie (ca. $26 \%$ nach 10 Tagen) entwickeln im weiteren Verlauf ein Viertel (CI95 16-32\%) eine symptomatische Harnwegsinfektion [12]. Allerdings kann diese CA-UTIRate in Abhängigkeit von Risikofaktoren der jeweiligen Patientenpopulation auch deutlich höher sein, insbesondere, wenn das Immunsystem der Patienten nachhaltig beeinträchtigt ist (z. B. unter einer zytostatischen Chemotherapie). Das Problem ist auch keineswegs auf die Intensivmedizin begrenzt. Vonberg et al. [27] untersuchten Deviceanwendungsraten und deviceassoziierte NI bei erwachsenen Patienten einer Universitätsklinik auch außerhalb von Intensivstationen. An 14,8\% aller Patiententage hatten die Patienten einen Harnwegskatheter, die Infektionsrate lag bei 6,8 pro 1000 Anwendungstage.

\section{Sekundäre Sepsis ausgehend von einer Infektion der Harnwege}

Katheterassoziierte Harnwegsinfektionen (CA-UTI) können mittelbar (z. B. durch Verschlechterung der Nierenfunktion bei Beteiligung des Nierenparenchyms) erhebliche negative Konsequenzen für die Patienten nach sich ziehen. In seltenen Fällen (insgesamt wahrscheinlich bei 3-4\% der Patienten $[12,28]$ ) kommt es im Verlauf einer CA-UTI zu einer sekundären Bakteriämie bzw. zu einer Urosepsis, bei der die Harnwegsinfektion der primäre Infektionsfokus ist. Auch wenn dieser Anteil von CA-UTI mit Urosepsis auf den ersten Blick niedrig erscheint, handelt es sich insgesamt - nach den gefäßkatheterassoziierten Blutstrominfektionen - um die zweithäufigste Form der nosokomialen Sepsis (14,8\% bei Melzer et al. 2013, $21 \%$ bei Fortin et al. 2012 [29, 30]). Coellho et al. [31] analysierten in einer Studie aus Großbritannien Blutstrominfektionen (BSI), die von einem Device ausgingen. Bei den deviceassoziierten BSI, die außerhalb von Universitätskliniken und Lehrkrankenhäusern diagnostiziert wurden ( $n=2103)$, stammten $61 \%$ aus den klinischen Gebieten Allgemeinmedizin, Allgemeinchirurgie, Geriatrie und Urologie. In der Geriatrie entfiel auf die mit einem zentralen Venenkatheter assoziierten BSI nur ein Anteil von 1,7\%, während die HWK-assoziierten Infektionen mit sekundärer BSI $21 \%$ aller deviceassoziierten Bakteriämien ausmachten. Ohne dass dieses Problem hier umfassend besprochen werden kann [23, 32-35], soll auf einige Aspekte besonders hingewiesen werden. Im Unterschied zur gefäßkatheterassoziierten Sepsis dominieren bei der Sepsis, die von einer Harnwegsinfektion (mit oder ohne Katheter) ausgeht, die gramnegativen Infektionserreger, vor allem E. coli, Klebsiella spp., Pseudomonas aeruginosa, seltener Proteus spp. Bei den grampositiven Erregern überwiegen die Enterokokken (inklusive vancomycinresistenter Enterococcus faecium [34, 36]), seltener auch S. aureus (inkl. MRSA [30]). Ein erheblicher Anteil [29, 37] der bei einer HWK-assoziierten Sepsis nachgewiesenen gramnegativen Infektionserreger erfüllt MRGN-Kriterien [38]. Dies gilt ins- besondere für Patienten mit Langzeitkatheter (Liegedauer >30 Tage) und multipler antibiotischer Vorbehandlung [39-41].

Unter anderem aufgrund des sehr hohen Durchschnittalters und begleitender Komorbiditäten kann die mit der nosokomialen Urosepsis verbundene Mortalität hoch sein. Zum Beispiel waren bei Melzer et al. 7 Tage nach Diagnose einer katheterassoziierten Urosepsis $30 \%$ (von 83 Patienten) und nach 30 Tagen $40 \%$ der Patienten verstorben [29]. In weiteren Studien [42] lag die Letalität der Patienten zwischen 9\% [41] und 33\% [34]. Die Therapie von komplizierten Harnwegsinfektionen durch MRGN stellt für die behandelnden Ärzte eine große Herausforderung dar [43].

\section{Besondere Bedeutung von E. coli}

Der besondere Stellenwert von E. coli als Erreger einer sekundären Bakteriämie im Kontext von Harnwegsinfektionen und deutlich seltener - auch von primär biliären Infektionen hat in Großbritannien zu einer Meldepflicht für BSI geführt, die durch E. coli verursacht werden [44, 45]. Auch aktuelle Publikationen aus den KISS-Modulen weisen auf die besondere Bedeutung und auf regionale Unterschiede in der Inzidenz von nosokomialen Infektionen durch ESBL-bildende E. coli hin $[46,47]$. Bei den ambulant erworbenen Harnwegsinfektionen nimmt der Anteil von E. coli mit MRGN-Eigenschaften zu [48]. Außerdem werden immer häufiger bestimmte, besonders gut adaptierte "pandemische" ESBL-bildende E.-coliIsolate nachgewiesen (z. B. E. coli O25:H4ST131 CTX-M-15) [49-51].

Diese Stämme können offenbar von Mensch zu Mensch übertragen werden [52] und sind auch in der industriellen Tiermast oder bei Haustieren (companion animals) nachgewiesen worden [53, 54]. Hierdurch kommt es bei den schweren Harnwegsinfektionen (mit und ohne Harnwegskatheter), die eine stationäre Therapie erforderlich machen, zu einem unerwünschten Eintrag bestimmter Erreger ins Krankenhaus, über deren nosokomiale Epidemiologie noch zu wenig bekannt ist $[46,47]$.

Die überarbeitete Empfehlung zur Prävention harnwegskatheterassoziierter In- 
fektionen soll in diesem komplexen klinisch-infektiologischen Kontext auch weiterhin als hilfreiche Handlungsanweisung zur Prävention von nosokomialen Infektionen und zur Eindämmung multiresistenter Infektionserreger dienen. Die aktuelle Überarbeitung zeigte, dass viele der 1999 gegebenen Empfehlungen bis heute weiterhin gelten; nichtsdestotrotz war es notwendig, dies erneut systematisch unter Berücksichtigung der neuesten Literatur zu überprüfen.

\section{Korrespondenzadresse}

\section{Prof. Dr. med. A. Simon}

Klinik für Pädiatrische Onkologie und

Hämatologie

Universitätsklinikum des Saarlandes, Kirrberger Straße, Gebäude 9, 66421 Homburg/Saar Arne.Simon@uks.eu

Interessenkonflikt. A. Simon gibt an, dass kein Interessenkonflikt besteht.

\section{Literatur}

1. Kommission für Krankenhaushygiene und Infektionsprävention beim Robert Koch-Institut B (1999) Empfehlungen zur Prävention und Kontrolle katheterassoziierter Harnwegsinfektionen. Bundesgesundheitsblatt Gesundheitsforschung Gesundheitsschutz 42:806-809

2. Fakih MG, Watson SR, Greene MT, Kennedy EH, Olmsted RN, Krein SL, Saint S (2012) Reducing inappropriate urinary catheter use: a statewide effort. Arch Intern Med 172:255-260

3. Halleberg Nyman M, Gustafsson M, Langius-Eklof A, Johansson JE, Norlin R, Hagberg L (2013) Intermittent versus indwelling urinary catheterisation in hip surgery patients: a randomised controlled trial with cost-effectiveness analysis. Int J Nurs Stud 50:1589-1598

4. Chenoweth C, Saint S (2013) Preventing catheterassociated urinary tract infections in the intensive care unit. Crit Care Clin 29:19-32

5. Crouzet J, Bertrand X, Venier AG, Badoz M, Husson C, Talon D (2007) Control of the duration of urinary catheterization: impact on catheter-associated urinary tract infection. J Hosp Infect 67:253-257

6. Alexaitis I, Broome B (2014) Implementation of a nurse-driven protocol to prevent catheter-associated urinary tract infections. J Nurs Care Qual 29:245-252

7. Palese A, Buchini S, Deroma L, Barbone F (2010) The effectiveness of the ultrasound bladder scanner in reducing urinary tract infections: a metaanalysis. J Clin Nurs 19:2970-2979

8. Parry MF, Grant B, Sestovic M (2013) Successful reduction in catheter-associated urinary tract infections: focus on nurse-directed catheter removal. Am J Infect Control 41:1178-1181

9. Reilly L, Sullivan P, Ninni S, Fochesto D, Williams K, Fetherman B (2006) Reducing foley catheter device days in an intensive care unit: using the evidence to change practice. AACN Adv Crit Care 17:272283
10. Titsworth WL, Hester J, Correia T, Reed R, Williams M, Guin P, Layon AJ, Archibald LK, Mocco J (2012) Reduction of catheter-associated urinary tract infections among patients in a neurological intensive care unit: a single institution's success. J Neurosurg 116:911-920

11. Cheerva AC, Raj A, Bertolone SJ, Bertolone K, Silverman CL (2007) BK virus-associated hemorrhagic cystitis in pediatric cancer patients receiving highdose cyclophosphamide. J Pediatr Hematol Oncol 29:617-621

12. Saint S (2000) Clinical and economic consequences of nosocomial catheter-related bacteriuria. Am J Infect Control 28:68-75

13. Fakih MG, Pena ME, Shemes S, Rey J, Berriel-Cass D, Szpunar SM, Savoy-Moore RT, Saravolatz LD (2010) Effect of establishing guidelines on appropriate urinary catheter placement. Acad Emerg Med 17:337-340

14. Gokula RM, Smith MA, Hickner J (2007) Emergency room staff education and use of a urinary catheter indication sheet improves appropriate use of foley catheters. Am J Infect Control 35:589-593

15. Gokula RR, Hickner JA, Smith MA (2004) Inappropriate use of urinary catheters in elderly patients at a midwestern community teaching hospital. Am J Infect Control 32:196-199

16. Wald H, Epstein A, Kramer A (2005) Extended use of indwelling urinary catheters in postoperative hip fracture patients. Med Care 43:1009-1017

17. Wald HL, Ma A, Bratzler DW, Kramer AM (2008) Indwelling urinary catheter use in the postoperative period: analysis of the national surgical infection prevention project data. Arch Surg 143:551-557

18. Blodgett TJ (2009) Reminder systems to reduce the duration of indwelling urinary catheters: a narrative review. Urol Nurs 29:369-378 (quiz 379)

19. Meddings J, Rogers MA, Krein SL, Fakih MG, Olmsted RN, Saint S (2013) Reducing unnecessary urinary catheter use and other strategies to prevent catheter-associated urinary tract infection: an integrative review. BMJ Qual Saf 23:277-289

20. Wald HL, Kramer AM (2011) Feasibility of audit and feedback to reduce postoperative urinary catheter duration. J Hosp Med 6:183-189

21. Hartley S, Valley S, Kuhn L, Washer LL, Gandhi T, Meddings J, Chenoweth C, Malani AN, Saint S, Srinivasan A, Flanders SA (2013) Inappropriate testing for urinary tract infection in hospitalized patients: an opportunity for improvement. Infect Control Hosp Epidemiol 34:1204-1207

22. Tambyah PA, Maki DG (2000) The relationship between pyuria and infection in patients with indwelling urinary catheters: a prospective study of 761 patients. Arch Intern Med 160:673-677

23. Trautner BW (2010) Management of catheter-associated urinary tract infection. Curr Opin Infect Dis 23:76-82

24. Trautner BW, Petersen NJ, Hysong SJ, Horwitz D, Kelly PA, Naik AD (2014) Overtreatment of asymptomatic bacteriuria: identifying provider barriers to evidence-based care. Am J Infect Control 42:653658

25. Blakiston M, Zaman S (2014) Nosocomial bacteriuria in elderly inpatients may be leading to considerable antibiotic overuse: an audit of current management practice in a secondary level care hospital in New Zealand. Infect Drug Resist 7:301-308

26. Cope M, Cevallos ME, Cadle RM, Darouiche RO, Musher DM, Trautner BW (2009) Inappropriate treatment of catheter-associated asymptomatic bacteriuria in a tertiary care hospital. Clin Infect Dis 48:1182-1188
27. Vonberg RP, Behnke M, Geffers C, Sohr D, Ruden H, Dettenkofer M, Gastmeier P (2006) Device-associated infection rates for non-intensive care unit patients. Infect Control Hosp Epidemiol 27:357-361

28. Tambyah PA, Maki DG (2000) Catheter-associated urinary tract infection is rarely symptomatic: a prospective study of 1,497 catheterized patients. Arch Intern Med 160:678-682

29. Melzer M, Welch C (2013) Outcomes in UK patients with hospital-acquired bacteraemia and the risk of catheter-associated urinary tract infections. Postgrad Med J 89:329-334

30. Fortin E, Rocher I, Frenette C, Tremblay C, Quach C (2012) Healthcare-associated bloodstream infections secondary to a urinary focus: the Quebec provincial surveillance results. Infect Control Hosp Epidemiol 33:456-462

31. Coello R, Charlett A, Ward V, Wilson J, Pearson A, Sedgwick J, Borriello P (2003) Device-related sources of bacteraemia in English hospitals-opportunities for the prevention of hospital-acquired bacteraemia. J Hosp Infect 53:46-57

32. Greene MT, Chang R, Kuhn L, Rogers MA, Chenoweth CE, Shuman E, Saint S (2012) Predictors of hospital-acquired urinary tract-related bloodstream infection. Infect Control Hosp Epidemiol 33:1001-1007

33. Saint S, Kaufman SR, Rogers MA, Baker PD, Boyko EJ, Lipsky BA (2006) Risk factors for nosocomial urinary tract-related bacteremia: a case-control study. Am J Infect Control 34:401-407

34. Chang R, Greene MT, Chenoweth CE, Kuhn L, Shuman E, Rogers MA, Saint S (2011) Epidemiology of hospital-acquired urinary tract-related bloodstream infection at a university hospital. Infect Control Hosp Epidemiol 32:1127-1129

35. Lee H, Lee YS, Jeong R, Kim YJ, Ahn S (2014) Predictive factors of bacteremia in patients with febrile urinary tract infection: an experience at a tertiary care center. Infection 42:669-674

36. Gastmeier P, Schroder C, Behnke M, Meyer E, Geffers C (2014) Dramatic increase in vancomycin-resistant enterococci in Germany. J Antimicrob Chemother 69:1660-1664

37. Sader HS, Flamm RK, Jones RN (2014) Frequency of occurrence and antimicrobial susceptibility of Gram-negative bacteremia isolates in patients with urinary tract infection: results from United States and European hospitals (2009-2011). J Chemother 26:133-138

38. Kommission für Krankenhaushygiene und Infektionsprävention beim Robert Koch-Institut B (2011) Definition der Multiresistenz gegenüber Antibiotika bei gramnegativen Stäbchen im Hinblick auf Maßnahmen zur Vermeidung der Weiterverbreitung. Epidemiol Bulletin des Robert Koch-Instituts, Berlin 12. September 2011:337-339

39. Moro ML, Ricchizzi E, Morsillo F, Marchi M, Puro V, Zotti CM, Prato R, Privitera G, Poli A, Mura I, Fedeli $U$ (2013) Infections and antimicrobial resistance in long term care facilities: a national prevalence study. Ann lg 25:109-118

40. Mylotte JM (2005) Nursing home-acquired bloodstream infection. Infect Control Hosp Epidemiol 26:833-837

41. Ortega M, Marco F, Soriano A, Almela M, Martinez JA, Pitart C, Mensa J (2013) Epidemiology and prognostic determinants of bacteraemic catheteracquired urinary tract infection in a single institution from 1991 to 2010. J Infect 67:282-287 


\section{Editorial}

42. Bishara J, Leibovici L, Huminer D, Drucker M, Samra Z, Konisberger H, Pitlik S (1997) Five-year prospective study of bacteraemic urinary tract infection in a single institution. Eur J Clin Microbiol Infect Dis 16:563-567

43. Pallett A, Hand K (2010) Complicated urinary tract infections: practical solutions for the treatment of multiresistant Gram-negative bacteria. J Antimicrob Chemother 65(Suppl 3):iii25-iii33

44. Melzer M, Welch C (2012) Is Escherichia coli bacteraemia preventable? Lancet Infect Dis 12:103-104

45. Underwood J, Klein JL, Newsholme W (2011)

Escherichia coli bacteraemia: how preventable is it? J Hosp Infect 79:364-365

46. Leistner R, Sakellariou C, Gurntke S, Kola A, Steinmetz I, Kohler C, Pfeifer Y, Eller C, Gastmeier P, Schwab F (2014) Mortality and molecular epidemiology associated with extended-spectrum beta-lactamase production in Escherichia coli from bloodstream infection. Infect Drug Resist 7:57-62

47. Leistner R, Schroder C, Geffers C, Breier AC, Gastmeier $P$, Behnke $M$ (2014) Regional distribution of nosocomial infections due to ESBL-positive Enterobacteriaceae in Germany: data from the German National Reference Center for the Surveillance of Nosocomial Infections (KISS). Clin Microbiol Infect 21:255.e1-5

48. Kresken $M$, Pfeifer Y, Hafner D, Wresch R, Korber-Irrgang $B$ (2014) Occurrence of multidrug resistance to oral antibiotics among Escherichia coli urine isolates from outpatient departments in Germany: extended-spectrum beta-lactamases and the role of fosfomycin. Int J Antimicrob Agents 44:295-300

49. Johnson JR, Johnston B, Clabots C, Kuskowski MA, Castanheira M (2010) Escherichia coli sequence type ST131 as the major cause of serious multidrugresistant E. coli infections in the United States. Clin Infect Dis 51:286-294

50. Morgand M, Vimont S, Bleibtreu A, Boyd A, Thien HV, Zahar JR, Denamur E, Arlet G (2014) Extendedspectrum beta-lactamase-producing Escherichia coli infections in children: are community-acquired strains different from nosocomial strains? Int J Med Microbiol 304:970-976

51. Clermont $\mathrm{O}$, Lavollay M, Vimont S, Deschamps $C$, Forestier C, Branger C, Denamur E, Arlet G (2008) The CTX-M-15-producing Escherichia coli diffusing clone belongs to a highly virulent B2 phylogenetic subgroup. J Antimicrob Chemother 61:1024-1028

52. Johnson JR, Anderson JT, Clabots C, Johnston B, Cooperstock M (2010) Within-household sharing of a fluoroquinolone-resistant Escherichia coli sequence type ST131 strain causing pediatric osteoarticular infection. Pediatr Infect Dis J 29:473475

53. Ewers $C$, Grobbel M, Stamm I, Kopp PA, Diehl I, Semmler T, Fruth A, Beutlich J, Guerra B, Wieler LH, Guenther S (2010) Emergence of human pandemic 025:H4-ST131 CTX-M-15 extended-spectrum-beta-lactamase-producing Escherichia coli among companion animals. J Antimicrob Chemother 65:651-660

54. Platell JL, Cobbold RN, Johnson JR, Trott DJ (2010) Clonal group distribution of fluoroquinolone-resistant Escherichia coli among humans and companion animals in Australia. J Antimicrob Chemother 65:1936-1938 